\title{
Indications of shock waves in the solar photosphere
}

\author{
J. Rybák ${ }^{1}$, H. Wöhl ${ }^{2}$, A. Kučera ${ }^{1}$, A. Hanslmeier ${ }^{3}$, and O. Steiner ${ }^{2}$ \\ 1 Astronomical Institute of the Slovak Academy of Sciences, 05960 Tatranská Lomnica, Slovakia \\ 2 Kiepenheuer-Institut für Sonnenphysik, Schöneckstr. 6, 79104 Freiburg, Germany \\ 3 Institut für Geophysik, Astrophysik und Meteorologie, Universitätsplatz 5, 8010 Graz, Austria \\ Received 11 November 2003 / Accepted 15 March 2004
}

\begin{abstract}
High resolution observations of solar granulation near the solar limb are used in a search for hydrodynamic shocks caused by an abrupt braking of the fast (probably supersonic) horizontal flow of the granular plasma towards the intergranular lane. Shock signatures in the spectral line of Fe II $6456.38 \AA$ of one particular observed shock event are investigated in detail. Evolution, amplitude, and spatial relation of the spectral line characteristics of the shock event are in agreement with predictions from numerical simulations for such shock phenomena in the solar photosphere. The dimensions and amplitudes of the observed shock signatures are comparable to predicted values when seeing and instrumental effects as well as a possible obliqueness of the shock front with respect to the observer's line-of-sight are taken into account. The temporal evolution of such an event is observed for the first time. The stable and declining phase of the event were studied for a time period of almost 2 min. A particular relationship was found between the shock event and a nearby $G$-band bright point located 2" from the shock event. It is suggestive that the observed shock is a causal consequence of the magnetic flux concentration, traced by the $G$-band bright point. Such a type of shock can appear outside the flux concentrations as a consequence of a rapid flux-tube motion.
\end{abstract}

Key words. Sun: photosphere - Sun: granulation - line: profiles - shock waves

\section{Introduction}

In the past two decades it has become feasible to study the nonstationary nature of the photospheric layers with the help of numerical simulations. First comprehensive simulations treated non-magnetic solar granulation (e.g., Nordlund 1984; Stein \& Nordlund 1989; Steffen et al. 1989). While these simulations included a sophisticated treatment of radiative transfer and subgrid turbulence models, other numerical work included less physics but concentrated on a detailed treatment of turbulence, both in 2D and 3D (e.g., Cattaneo et al. 1989, 1990; Malagoli et al. 1990). Simulations of convective motion including magnetic field have been carried out in 3D by Nordlund (1986) and Weiss et al. (1996) and more recently by Cattaneo (1999), Emonet \& Cattaneo (2001), Stein \& Nordlund (2002) or Vögler $\&$ Schüssler (2003) and in 2D in a series of papers by Deinzer et al. (1984a,b), Knölker et al. (1988), Grossman-Doerth et al. (1988, 1994), and by Hurlburt \& Toomre (1988), Fox et al. (1999), and Ploner et al. (1999). Steiner et al. (1998) simulated the dynamical interaction of a preexisting magnetic flux sheet with a fully developed granular convection while Grossman-Doerth et al. (1988) studied the formation of flux sheets in a non-stationary convective environment. The latter two works include the computation of synthetic spectra from simulation snapshots thus enabling the prediction of spectral features, which can be looked for in observed high spatial and spectral resolution spectra of solar granulation.

Send offprint requests to: J. Rybák, e-mail: choc@astro.sk
Simulations have predicted supersonic horizontal flows of granular plasma as a consequence of the non-adiabatic cooling at the solar surface. The collision of the horizontal flow of neighbouring granules leads to the formation of a pair of shocks which travel from the intergranular lane upstream toward the granule centres (Cattaneo et al. 1989, 1990; Malagoli et al. 1990; Steffen \& Freytag 1991; Steiner et al. 1994; Solanki et al. 1996; Gadun 2000). The first attempt to confirm the existence of these horizontally moving shocks was made by Nesis et al. (1992). The observational evidence for such flows in the intergranular lanes was based on the negative correlation between the line width and the continuum intensity. They argued that the excess in line width was caused by turbulence introduced by the shock that brakes the supersonic flow back to a subsonic one in the intergranular lane. Later, Nesis et al. (1993) found the region of the enhanced turbulence to be concentrated at the borders of granules, especially when there exists a steep velocity gradient. High resolution observations made by Solanki et al. (1996) qualitatively confirmed the trend of the relation found by Nesis et al. (1992). On the other hand Solanki et al. (1996) showed, based on numerical simulations, that spectral lines that originate in the intergranular lanes are broader even in the absence of any horizontal supersonic flow and associated shock front. The latter however seem to be prerequisite for the line broadening when observed near the solar limb. The direct spatial identification of enhanced line broadening at the boundaries of some granules, supports the interpretation of the line broadening in terms of shocks that are formed near granular 
Table 1. Atomic parameters and height of formation of the Fe II lines selected for observations of shock signatures. $E_{\mathrm{p}}$ and $W_{\lambda}$ stand for the excitation potential of the lower level and the equivalent width of the line, respectively. The Landé factors are given for the cases of LS coupling and the empirical values. The height of formation was calculated according to the MACKKL model of the solar photosphere (Maltby et al. (1986) by Gadun (2000). References: 1 - Allende Prieto \& Garciá López (1998); 2 - Moore (1972); 3 - Kurucz (1990); 4 - Solanki \& Stenflo (1985); 5 - Moore et al. (1966).

\begin{tabular}{ccccccc}
\hline \hline Solar Wavelength $^{1}[\AA]$ & Multiplet $^{2}$ & $E_{\mathrm{p}}{ }^{3}[\mathrm{eV}]$ & Transition $^{4}$ & $W_{\lambda}{ }^{5}[\mathrm{~m} \AA]$ & Landé factor $^{4}$ & Formation height $[\mathrm{km}]^{2}$ \\
\hline 4993.3527 & 36 & 2.807 & $\mathrm{~b}^{4} \mathrm{~F}_{4 \frac{1}{2}}-\mathrm{z}^{6} \mathrm{P}_{3 \frac{1}{2}}$ & 34 & $0.667 / 0.616$ & 110 \\
6456.3888 & 74 & 3.903 & $\mathrm{~b}^{4} \mathrm{D}_{3 \frac{1}{2}}-\mathrm{z}^{4} \mathrm{P}_{2 \frac{1}{2}}$ & 57 & $1.214 / 1.182$ & 205 \\
\hline
\end{tabular}

boundaries as a consequence of the colliding horizontal granular flow.

Steiner et al. (1998) detected three types of shocks in 2D MHD simulations of a magnetic flux sheet in interaction with granulation depending on the place of origin and direction of propagation. Type I shocks belong to the deceleration zone of the horizontal granular flow on the edge of the intergranular lane. This type is in agreement with the previously predicted shock from non-magnetic simulations made by Cattaneo et al. (1989, 1990) and other authors and is not related to the presence of the flux sheet. Type II shocks are caused by the swaying motion of the flux sheet when a fast motion of the sheet pushes the plasma in front of it creating a vertically oriented bow shock moving horizontally. Type III shocks are found to be horizontally oriented and propagating upward within the flux sheet itself. This type is generated by the deformation of the flux sheet and tube-mode coupling due to the dynamics of convection.

Each of the three types of shocks can be expected to produce signatures in spectral lines. In order to distinguish them their temporal evolution, direction of propagation, and their location with respect to the granular/intergranular intensity and the magnetic pattern must be determined. As we concentrate on horizontal flows in the following, type III shocks are not considered. The aim of this paper is to give direct evidence for shocks in the solar photosphere including their temporal development using high spatial and spectral resolution observations of the photospheric lines, given in Table 1. A preliminary report was given by Kučera et al. (2003) ${ }^{1}$. Predictions of the shock signatures from the numerical simulations are summarized in Sect. 2 and our observations are described in Sects. 3 and 4. Results on the observed spectral signatures of the suspected shock events are given in Sect. 5. Discussion of the results and a comparison of the observed shock signatures to the $G$-band intensity pattern and to synthetic spectral line profiles are presented in Sect. 6. Final conclusions are provided in Sect. 7.

\section{Predictions from numerical simulations}

Radiative transfer analysis of numerical simulations predict distinctive signatures of spectral profiles when a shock travels

${ }^{1}$ Due to a program error the full line width given in this report was divided by a factor of two. In addition the equivalent width of the investigated spectral line was given as 81 instead of $57 \mathrm{~m} \AA$ (Table I of that paper). through the formation region of the spectral line in the photosphere. These shock signatures can be summarized as follows:

- A significant increase of the line width up to $300 \%$ of its average value when no degradation due to atmospheric and instrumental effects is taken into account (Gadun 2000; Gadun \& Hanslmeier 2000) or up to $100 \%$ after degradation to 0.5" spatial resolution (Solanki et al. 1996). Observations near the limb rule out the possibility that the same effect can be caused by the downward motion in the intergranular lane (Solanki et al. 1996).

- An increase of the residual line intensity as the shock heats the post-shock plasma. Increase of the residual intensity caused by the shock was found to be $250 \%$ of its unaffected value for calculations without degradation and $75 \%$ after artificial degradation due to the expected seeing and instrumental effects. This provides one possible explanation for the line gap phenomenon - a strong weakening of spectral lines - studied for example by Steffen et al. (1994) and Steiner et al. (1998) although other mechanisms caused by the magnetic field have been presented too (Amer \& Kneer 1993).

Similar results were found by Gadun \& Hanslmeier (2000) who also showed that the relative position of shock signatures in the line width and in the residual line intensity changes depending on the inclination of the line-of-sight with respect to the shock orientation.

- Simulations have predicted extreme jumps of line shift due to jumps of velocity across the shock of greater than $5 \mathrm{~km} \mathrm{~s}^{-1}$ (Solanki et al. 1996; Steiner et al. 1998; Gadun \& Hanslmeier 2000). A weakening and broadening of jump signatures can be expected due to the finite spatial resolution of the instrument and seeing conditions as well as due to the specific orientation of the shock relative to the line-of-sight of the observations. Both signs of the velocity are expected depending on the direction of the shock propagation. Jumps in line shifts could not be detected using low spatial resolution or when the shock front is not close to perpendicular with respect to the line-of-sight (Solanki et al. 1996).

- Only very small or no change of the continuum intensity caused by shocks was found (Steffen et al. 1994).

- Shocks cause only small changes in equivalent width of spectral lines, since an increase of the line width is roughly compensated for by an increase of the residual intensity. 
- Individual line profiles derived from simulations show extremely distorted or even double line profiles (Solanki et al. 1996; Steiner et al. 1998). Nevertheless the effect of finite spatial resolution and seeing, etc., smooths synthesized spectral line profiles (an illustrative example is given by Steiner et al. 1998). The actual shape of the observed spectral profile also depends on the orientation of the shock front with respect to the line-of-sight.

- The line profiles synthesized by Solanki et al. (1996) from numerical simulations made by Steffen \& Freytag (1991) have shown that near the solar limb the intergranular lanes do not show any significant broadening due to the downflow present there, in contrast to what is observed at disk centre. Therefore, observations made at large viewing angles should be free from the ambivalence of the enhanced line broadening introduced by the downflow in the intergranular lanes.

Results of Gadun \& Hanslmeier (2000) based on the simulations performed by Gadun et al. (1999) also suggest to carry out observations of shocks near the limb because of the higher chance there to find corresponding changes in spectral line characteristics. Additionally, he found a higher sensitivity of lines of Fe II to photospheric shocks compared to lines of the neutral atoms. The actual viewing angle under which the shock is observed in three-dimensional space is found to be important. Almost no effect on the spectral profiles takes place if the shock is viewed from a direction parallel to the shock front or from a direction oblique to that (Solanki et al. 1996). Generally, observing perpendicular to the shock front strongly favours registration of the shock in the spectral profile. Both, the synthesized line profiles calculated outside of the magnetic sheet by Steiner et al. (1998) and those profiles derived from the HD simulations (Steffen et al. 1994; Solanki et al. 1996; Gadun \& Hanslmeier 2000), have shown that line profiles can serve as a diagnostic of shocks. Very high spatial resolution is required, however.

\section{Observations}

A careful selection of Fe II lines in the visible wavelength range of the spectrum was performed. The amplitude of the expected shock signatures in the Fe II spectral line profile (Gadun 2000; Gadun \& Hanslmeier 2000), the formation heights, and other suitable lines near the chosen Fe II lines as well as the presence of any line blends were considered. Finally, only two Fe II lines (4993.35 $\AA$ and $6456.38 \AA$ ) were selected from eight suitable lines when we also considered the limitations of the spectrograph and our aim to measure at least two different spectral regions simultaneously in order to increase the number of measured lines (Table 1). These lines probe intermediate layers of the photosphere.

Observations were carried out with the Vacuum Tower Telescope (VTT) (Schröter et al. 1985) at the Observatorio del Teide, Tenerife, Spain. The Adaptive Optics (AO) of the National Solar Observatory at Sacramento Peak (Sunspot, USA) was allocated on loan for a test deployment at the VTT (Rimmele et al. 1999). The experimental setup of the AO was used successfully to compensate for pointing errors due to guiding inaccuracies and/or seeing for most of the observing time. The actual position of the spectrograph slit on the projected solar surface was recorded using the slit-jaw (SJ) imaging system (Kentischer 1995). In particular a $G$-band filter of $9 \AA$ bandpass centred at $4306 \AA$ was used for the slit-jaw image acquisition. A large Echelle spectrograph with the predisperser was used to produce high resolution spectra in two different spectral regions at the same time.

This paper is based on measurements taken on April 27, 2000 when two spectral regions (both about $3 \AA$ wide) around the selected Fe II lines were recorded. The spectrograph slit was oriented perpendicularly to the horizon to allow post-facto compensation for differential refraction. The width of the slit was equivalent to $0.5^{\prime \prime}$. The spectral data were acquired with two 12 bit XEDAR CCD cameras of $1 \mathrm{k} \times 1 \mathrm{k}$ frame. The spatial sampling was $0.125^{\prime \prime} /$ pixel and the spectral sampling was $2.14 \mathrm{~m} \AA /$ pixel and $3.09 \mathrm{~m} \AA /$ pixel for the first and second spectral region, respectively. The spectra were acquired simultaneously with an exposure time of $1 \mathrm{~s}$ and a dwell time of $3 \mathrm{~s}$. The SJ system was equipped with a $1 \mathrm{k} \times 1 \mathrm{k} 16$ bit DALSA digital camera operated with an exposure time of $5 \mathrm{~ms}$ and with a cadence of 2 frames per second. The spatial sampling was $0.107^{\prime \prime} /$ pixel.

The data were taken near the solar limb $(\mu=0.65)$ and a spot in the active region NOAA 8967 was used as a target for the AO tracking. The seeing conditions were of medium quality allowing for an estimated spatial resolution of about $0.5^{\prime \prime}$. Despite the fact the AO was used to track a relatively high intensity gradient (small spot) few intervals of lower contrast and also random motion of the solar image for a few tens of an arcsecond occurred during the measurements as can be seen from Fig. 1. Part of the slit crossed the penumbra of the spot and the rest of the slit covered the quiet atmosphere toward the limb. The slit was oriented perpendicular to the horizon, $50^{\circ}$ off the north-south direction. The whole observing run started at 10:20:54 UT and lasted for $15 \mathrm{~min}$.

\section{Data reduction}

The spectra were photometrically reduced over the $100^{\prime \prime}$ spatial distance along the slit. The precise reduction procedure (Wöhl et al. 2002) was applied. This procedure takes the temporal changes of the flat field conditions into account by splitting the flat-field matrix into two components - one stable in time connected to camera artefacts, the other one variable in time reflecting the long-term drift of the spectrograph. High frequency noise was removed from the individual spectra by optimal filtering (Gray 1976). The effects of filtering were carefully checked so as not to deform the profiles of the lines. The polynomial approximation of the dispersion was determined from the averaged disk centre spectra using the wavelength data of Moore et al. (1966). The zero point of the wavelength scale was adjusted according to the average positions of lines derived from all the spectra of the run. The intensity scale was normalized using the quasi-continua in the spectra taking into account their relation to the true continuum level (Neckel 1999). The centre-to-limb darkening of the continuum intensity was 

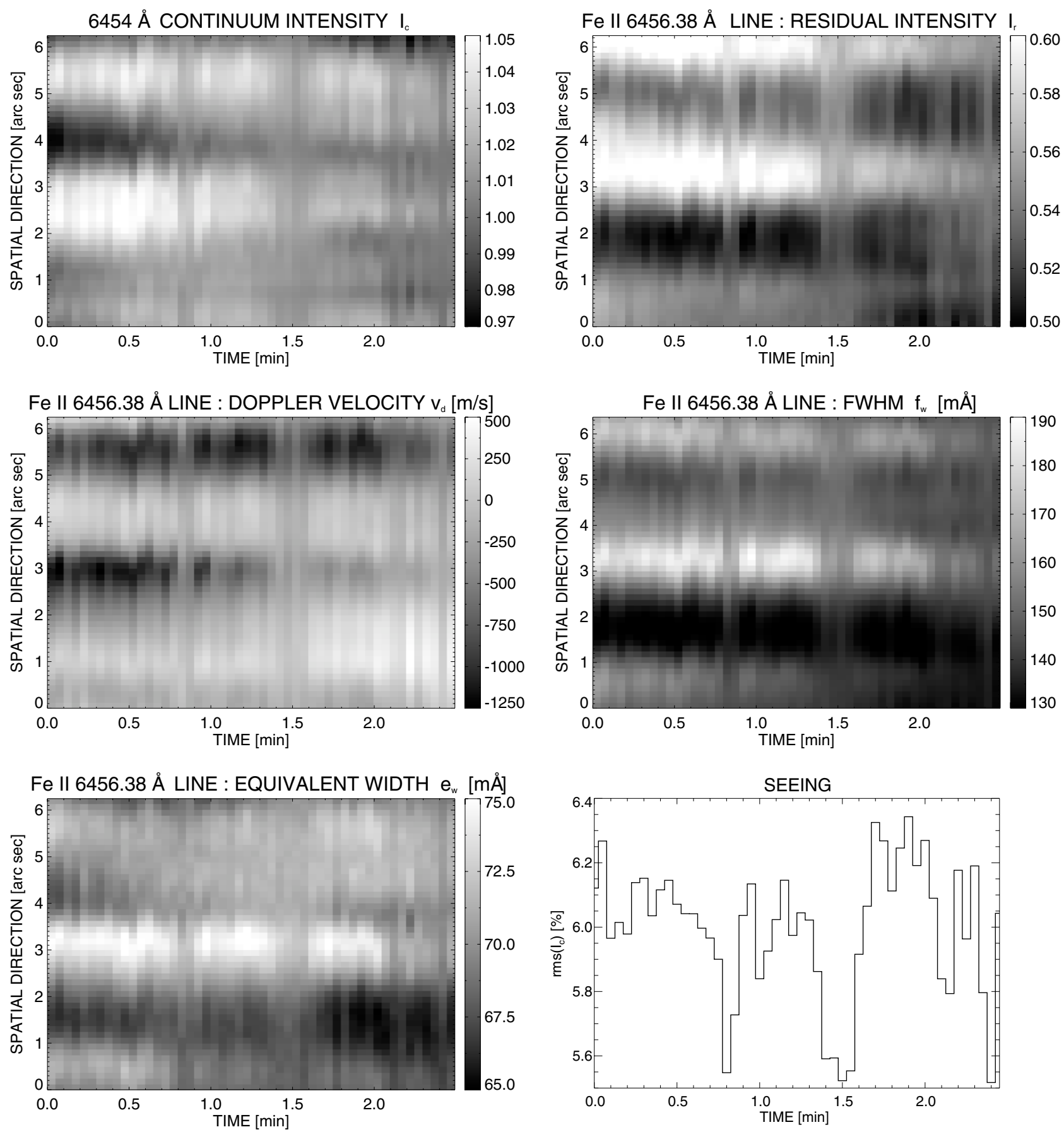

Fig. 1. Temporal and spatial behaviour $\left(2.5 \mathrm{~min} \times 6.3^{\prime \prime}\right)$ of the Fe II $6456.38 \AA$ A spectral line characteristics $I_{\mathrm{c}}, I_{\mathrm{r}}, v_{\mathrm{d}}, f_{\mathrm{w}}$, and $e_{\mathrm{w}}$ around the selected $f_{\mathrm{w}}$-event (position $3.3^{\prime \prime}$ ). No correction for the 5 min velocity oscillations was performed leaving the zero level of $v_{\mathrm{d}}$ uncertain to within several hundreds of $\mathrm{m} / \mathrm{s}$. The limb is in the downward direction of the plots. The spatial scale of the plots should be multiplied by a factor of 1.538 to obtain real distances on the solar surface. The actual seeing conditions traced from the temporal variations of the $I_{\mathrm{c}}$ rms-contrast are shown in the bottom right panel. The vertical structure in the plots is caused by different seeing quality. The image jitter at the entrance slits, caused by seeing, does not lead to a reduction of contrast of the spectral characteristics (e.g. around the time $0.5 \mathrm{~min}$ ). Occasional low spatial gradients of observed spectral characteristics are due to moments of bad observational conditions (e.g. as around 0.8 and $1.5 \mathrm{~min}$ ).

corrected for. The reference continuum intensity was calculated as the average value of the continua of all the spectra of the run. Each spectrum was normalized according to this reference continuum intensity. The following spectral characteristics were calculated for each spectral line in all the spectra of the run: $I_{\mathrm{c}}-$ the continuum intensity, $I_{\mathrm{r}}-$ the residual line intensity, $v_{\mathrm{d}}-$ the
Doppler velocity of the line, $f_{\mathrm{w}}$ - the full width at half depth, and $e_{\mathrm{w}}-$ the equivalent width of the line. The zero value of the Doppler velocity, $v_{\mathrm{d}}$, was derived from the mean spatial and temporal position of the line in the whole run when the convective blue shift and its centre-to-limb variation has been taken into account. Velocities toward the observer are negative. The 
equivalent widths have been calculated excluding the far wings to prevent contamination by blends.

The SJ images have also been photometrically reduced correcting for the mean dark current and the mean flat-field frames using the procedures provided by Langhans (2000). The narrow field of the SJ image along the slit was extracted $\left(6.2^{\prime \prime} \times 99.6^{\prime \prime}\right)$ and co-aligned with the spectra and the empirically determined differential refraction was corrected for. The SJ images acquired during the best seeing conditions were selected for comparison with the acquired spectra.

\section{Results}

According to the predictions of the numerical simulations a search for possible shock signatures was performed in that part of the data set pertaining to a quiet region atmosphere $\left(80^{\prime \prime} \times 15 \mathrm{~min}\right)$. The only signature used in this search was the line width, $f_{\mathrm{w}}$, of the Fe II $6456.38 \AA$ line. The line width was selected as it shows the most remarkable line signature of the shock as was demonstrated by the simulation of spectral profiles by Solanki et al. (1996), Steiner et al. (1998), and Gadun $\&$ Hanslmeier (2000). The line-width enhancement is produced by the large velocity gradient across the shock. In this paper only the results of the event with the most significant signature in line width are presented. This $f_{\mathrm{w}}$-event appeared at $\mu=0.62$ at a distance of $70^{\prime \prime}$ from the mentioned spot toward the limb.

The temporal and spatial behaviour of the spectral characteristics of the selected $f_{\mathrm{w}}$-event is shown in Fig. 1 . This event is located at $3.3^{\prime \prime}$ on the spatial scale of Fig. 1 and following figures. Only the stationary and declining phase of the selected $f_{\mathrm{w}}$-event can be followed as the onset phase of the event had taken place before the start of the observations. The average value of $I_{\mathrm{c}}$ was derived taking the limb-darkening into account. Representative spatial scans of the spectral characteristics for three temporal instances and for a reference moment are displayed in Fig. 2. From Figs. 1 and 2 the following properties and relations between the spectral characteristics of the $f_{\mathrm{w}}$-event can be derived:

- The event is confined to a spatial extent of just above $0.5^{\prime \prime}$ during its whole duration and it does not change position. The maximum $f_{\mathrm{w}}$-value is $194 \mathrm{~m} \AA$, i.e. $130 \%$ of the corresponding average value. The stationary phase of the event has a duration of about $1.3 \mathrm{~min}$ followed by a declining phase lasting for another $0.7 \mathrm{~min}$.

- The maximum values of the residual intensity, $I_{\mathrm{r}}$, are cospatial with the maximum values of $f_{\mathrm{w}}$. This co-spatiality can be seen despite the jitter along the slit due to the seeing. A residual intensity, $I_{\mathrm{r}}$, of up to $0.61 I_{\mathrm{c}}$ is measured, corresponding to a change of $11 \%$ relative to the average value of $I_{\mathrm{r}}$.

- With help of the continuum intensity, $I_{c}$, one can locate the event relative to the granular structure. Accordingly, the $f_{\mathrm{w}}$-event is located directly at the edge of the granule, where the gradient of $I_{\mathrm{c}}$ is largest. The granule has a continuum intensity that is $4 \%$ higher than the average $I_{\mathrm{c}}$ value. The intergranular lane seen near the $f_{\mathrm{w}}$-event (at about $4^{\prime \prime}$ ) as well as the granule itself seem to diminish in parallel with the evolution of the $f_{\mathrm{w}}$-event.

- Comparing the Doppler velocity, $v_{\mathrm{d}}$, with the variations of $f_{\mathrm{w}}$ it is found that the $f_{\mathrm{w}}$-event is not co-spatial with the extremes of the velocity but with the maximum value of the spatial gradient of $v_{\mathrm{d}}$ that is greater than $150 \mathrm{~m} \mathrm{~s}^{-1}$ over a distance of $0.1^{\prime \prime}$ (in the projection to the plane of sky). The maximum total difference of $v_{\mathrm{d}}$ across the event was found to be $1200 \mathrm{~m} \mathrm{~s}^{-1}$ over a distance of $1^{\prime \prime}$. At the centre of the $f_{\mathrm{w}}$-event a Doppler velocity of $-300 \mathrm{~m} \mathrm{~s}^{-1}$ (toward the observer and relatively to the zero-value defined in Sect. 4) was detected. Doppler velocities less then $+100 \mathrm{~m} \mathrm{~s}^{-1}$ (away from the observer) were found on the "disk centre side" (top-side in Fig. 1) of the $f_{\mathrm{w}}$-event. On the "limb side" of the $f_{\mathrm{w}}$-event, high $v_{\mathrm{d}}$-values of up to $-1200 \mathrm{~m} \mathrm{~s}^{-1}$ were measured. The latter value persists to the edge of the $f_{\mathrm{w}}$-event at the position of the highest spatial gradient of $f_{\mathrm{w}}$ on this side. The jump in velocity across the $f_{\mathrm{w}}$-event varied by more than $1000 \mathrm{~m} \mathrm{~s}^{-1}$ on a time scale less than $2.5 \mathrm{~min}$. It is worth to mention here that this jump cannot be a consequence of the seeing variation as the $I_{\mathrm{c}}$ rms-contrast stays at the same level most of the time (Fig. 1, bottom right panel). No correction for the $5 \mathrm{~min}$ velocity oscillations was performed. Therefore, the global shift of the zero velocity level can be affected by several hundreds of $\mathrm{m} / \mathrm{s}$ even at a particular position near the solar limb, and a large-scale trend in $v_{\mathrm{d}}$ cannot be ruled out. Nevertheless, the small-scale variation of $v_{\mathrm{d}}$ on the scale of an $1^{\prime \prime}$ is real.

- A small but clear increase of the equivalent line width, $e_{\mathrm{w}}$, of $4 \%$ of its average value was found to be co-spatial and co-temporal with the $f_{\mathrm{w}}$-event.

Besides the discussed $f_{\mathrm{w}}$-event a smaller event is located at position 5.8" (toward disk centre) that shows an increase of $f_{\mathrm{w}}$ by $16 \%$. Its position on the slit relative to other spectral characteristics is similar to the former event with the exception that no excess is found with regard to $e_{\mathrm{w}}$. Also, smaller amplitudes for $v_{\mathrm{d}}$ are measured for this event. Moreover the velocity amplitude does not decrease as drastically as in the former case towards the end of the event (different to the $v_{\mathrm{d}}$-amplitude difference of $1000 \mathrm{~m} \mathrm{~s}^{-1}$ in the former case). In the opposite direction (towards the limb) no enhancement of $f_{\mathrm{w}}$ is found along the slit over a distance of more than $10^{\prime \prime}$.

Besides the spectral characteristics, typical spectral profiles of the $f_{\mathrm{w}}$-event were examined together with a reference profile at the same centre-to-limb distance. These profiles of the Fe II $6456.38 \AA$ line and of two neighbouring lines of Ca I $6455.6 \AA$ and Co I $6455.0 \AA$ are shown in Fig. 3. The Fe II line profile of the $f_{\mathrm{w}}$-event shows, in addition to the already reported enhancement of $f_{\mathrm{w}}$, an increase of $I_{\mathrm{r}}$, and a significant blue shift. There is a clear line asymmetry in that the red wing of the profile is less steep. Moreover, the profiles of the $\mathrm{Ca}$ I and $\mathrm{Co}$ I lines display in all aspects a similar qualitative behaviour as was found for the Fe II line.

Examples of the $G$-band slit-jaw images acquired during the $f_{\mathrm{w}}$-event are shown in Fig. 4 . These SJ-images were prepared in a way that the $f_{\mathrm{w}}$-event is located in the middle of each panel, underneath the vertical dark slit at the position of $3.3^{\prime \prime}$. 
CONTINUUM INTENSITY I.

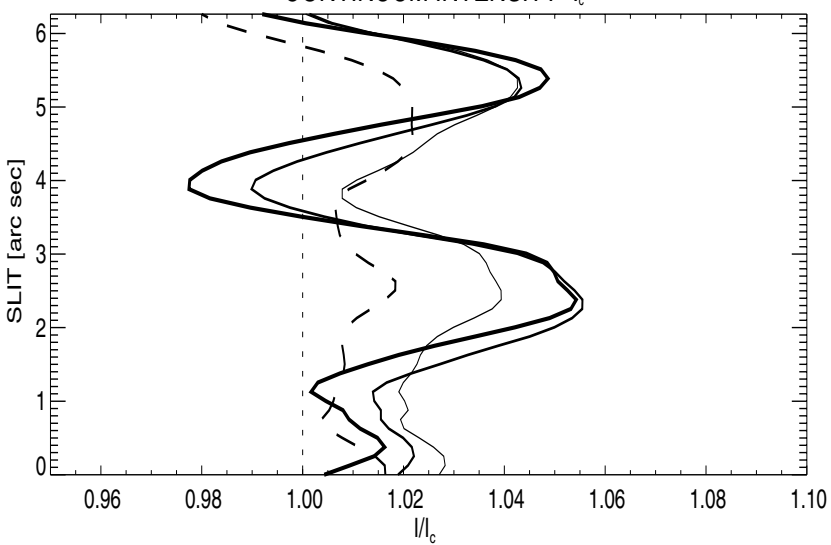

DOPPLER VELOCITY $V_{d}$

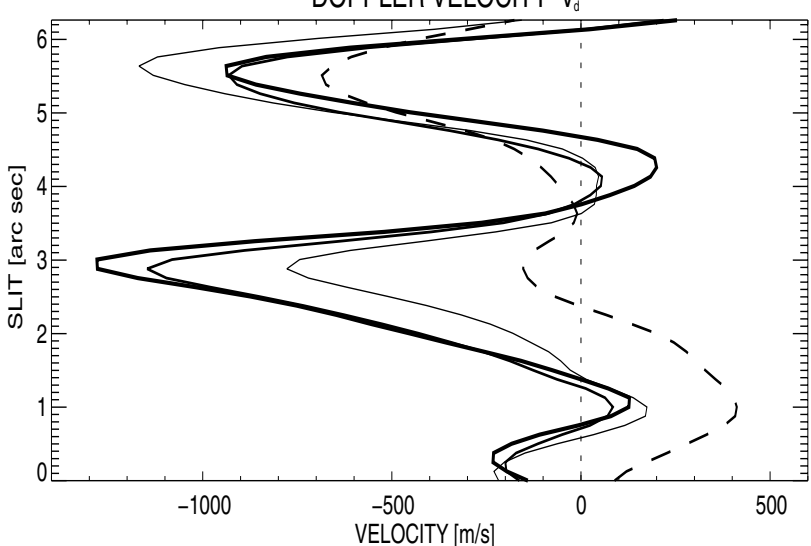

EQUIVALENT WIDTH $e_{w}$

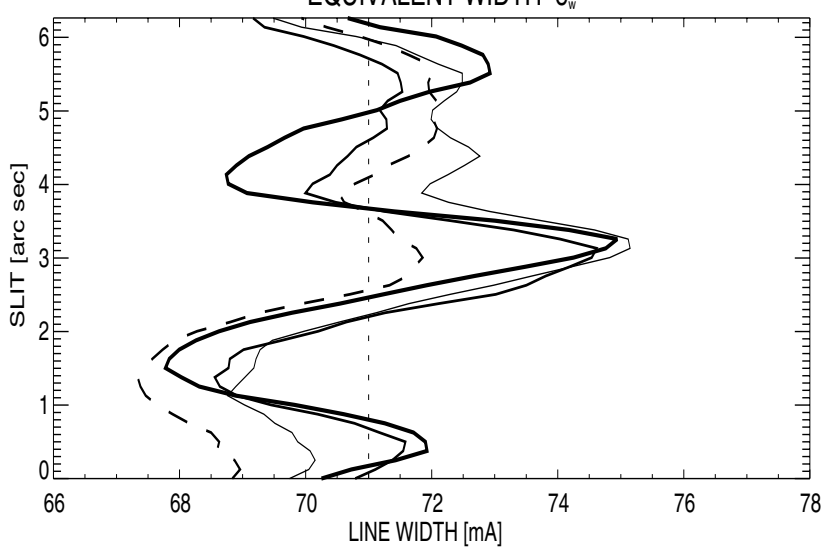

RESIDUAL INTENSITY I,

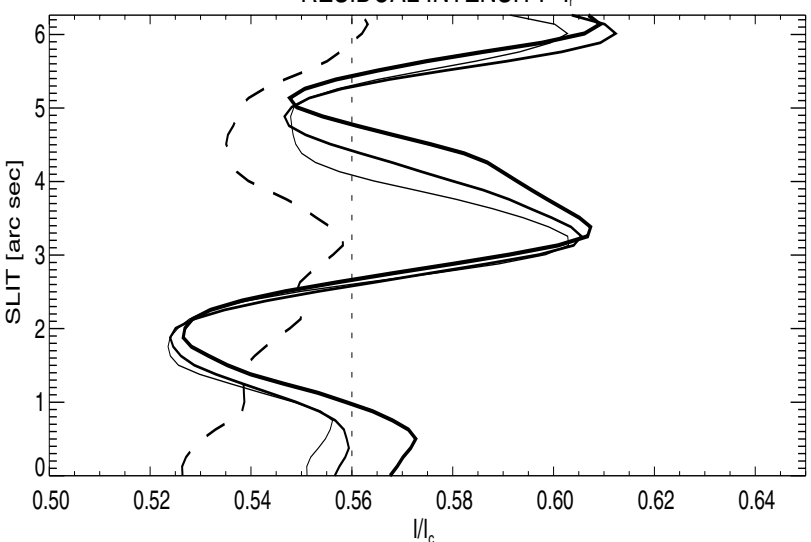

FWHM $f_{\text {w }}$

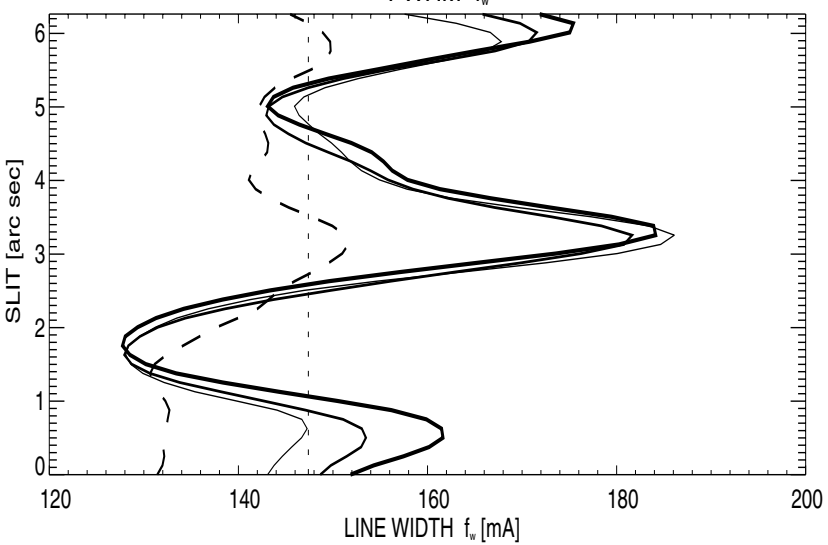

FWHM $f_{w}$

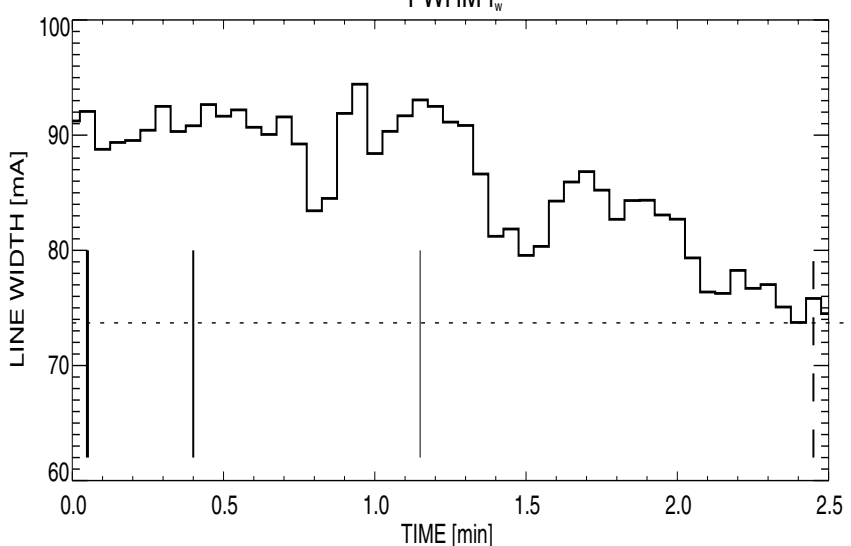

Fig. 2. Representative spatial scans of the Fe II $6456.38 \AA$ spectral characteristics for three time instances $(0.05,0.4$ and 1.16 min) during the event (solid lines of different thickness) and for a reference time (dashed line). The scans were extracted from Fig. 1. The temporal evolution of the line width, $f_{\mathrm{w}}$, of the considered $f_{\mathrm{w}}$-event is shown in the bottom right panel where the selected time instances are indicated by vertical lines of the corresponding line thickness used in the other panels. The solar limb is in the downward direction in all the plots. The spatial scale must be multiplied by a factor of 1.538 to get real distances on the solar surface. Dotted lines show the average values of the characteristics over a slit length of $80^{\prime \prime}$ and over a time of $15 \mathrm{~min}$.

The SJ-images show that besides a very weak regular granular pattern, there was a $G$-band bright point located very near the $f_{\mathrm{w}}$-event in the direction of the limb at $3.8^{\prime \prime}$ in the direction perpendicular to and $2.4^{\prime \prime}$ along the slit.

The intensity evolution of the $G$-band bright point is shown in Fig. 5. The bright point lasted for more than half a minute with roughly the same maximum intensity. During this period the intensity of the $G$-band bright point varied due to the variable seeing conditions from 1.10 to 1.18 relative to the mean $G$-band intensity. Then the intensity declines for $1.5 \mathrm{~min}$. An image of exceptionally high contrast, taken at $t=1.02$ min during the declining phase of the bright point, shows a bright point intensity of 1.19 , indicating that the high-intensity phase may have lasted for more than $1 \mathrm{~min}$ or that the previous maximum 

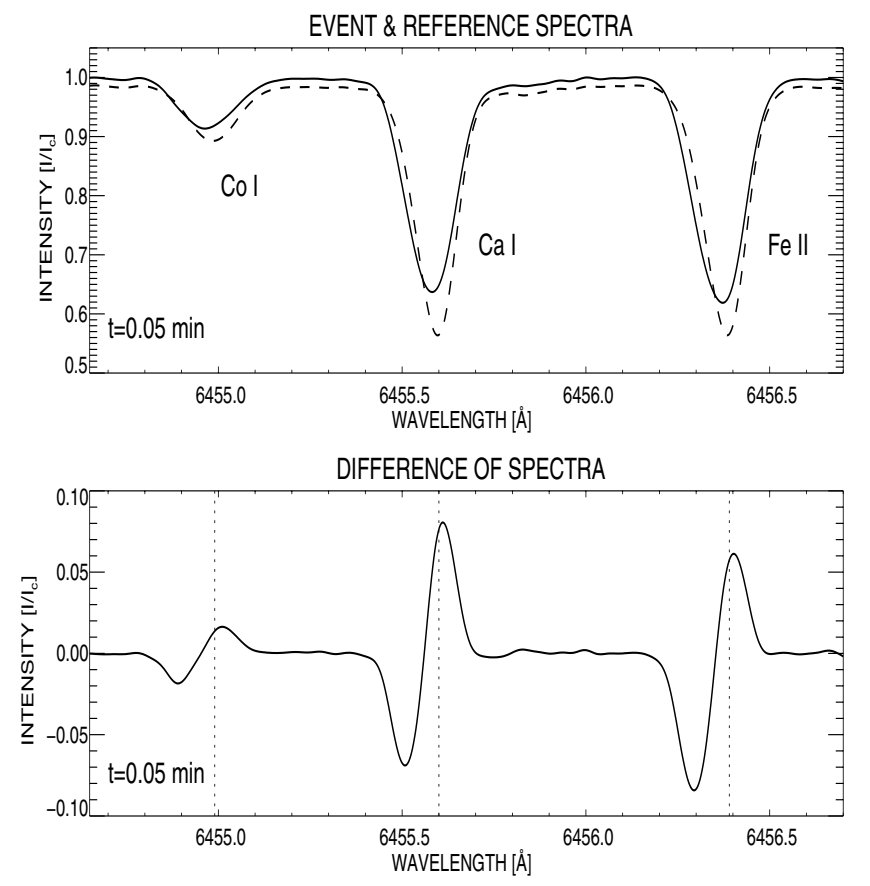

Fig. 3. Profiles of the CoI $6455.0 \AA$, Ca I $6455.6 \AA$, and Fe II $6456.38 \AA$ spectral lines of the $f_{\mathrm{w}}$-event. Upper panel: the line profiles taken during the $f_{\mathrm{w}}$-event $\left(t=0.05 \mathrm{~min}\right.$ at slit position $\left.3.3^{\prime \prime}\right)$ drawn with solid line together with the corresponding reference spectrum (dashed). The reference spectrum was prepared from spectra taken at the same centre-to-limb distance averaging several exposures around the time $t=2.5 \mathrm{~min}$. The relative difference of the spectra calculated with an identical continuum intensity is shown in the bottom panel. The vertical dashed lines mark the line centre positions of the reference spectrum.

bright point intensities (e.g., $t=0.07$ and $t=0.41 \mathrm{~min}$ ) could be even higher than 1.2. After two minutes, the bright point had diminished to the typical granular maximum intensity of the surrounding field.

The distance between the $f_{\mathrm{w}}$-event and the $G$-band bright point is $1.24 \pm 0.25^{\prime \prime}$ in the plane of sky corresponding to $2.08 \pm 0.43^{\prime \prime}$ on the solar surface. The mean value of this distance and its standard deviation were calculated from the distances of the bright point to the averaged $f_{\mathrm{w}}$-event position in all SJ-frames within the first minute of the measurement. The errors obtained here come from motions of the SJ-image caused by seeing. No systematic motion of the $f_{\mathrm{w}}$-event and the $G$-band bright point was detected under the particular seeing conditions. Comparing the evolution of the $G$-band bright point intensity and the changes of the line width, $f_{\mathrm{w}}$, during the $f_{\mathrm{w}}$-event, a constant phase is seen over the first minute of the measurement. In the second minute, a more rapid dimming of the $G$-band bright point intensity than the slower decline of $f_{\mathrm{w}}$ was observed.

\section{Discussion}

The selected $f_{\mathrm{w}}$-event fulfills the basic properties of a shock signature according to the predictions of numerical simulations obtained for shocks outside magnetic field concentrations (Sect. 1). The event is located at the border of a granule as marked by the largest gradient of the continuum intensity, $I_{\mathrm{c}}$. Its position can be clearly distinguished, both, from the granulum area and also from the middle of the intergranular lane (Figs. 1 and 2). The event is co-spatial with a significant increase of the residual line intensity, $I_{\mathrm{r}}$, indicating that the plasma inside the event is heated up above the border of the granulum. The largest gradient of the Doppler velocity, $v_{\mathrm{d}}$, is detected at the position of the event, with a maximum velocity difference of $1200 \mathrm{~m} \mathrm{~s}^{-1}$. The plasma moves toward the observer on the limb side of the $f_{\mathrm{w}}$-event with a velocity component along the line-of-sight of more than $1000 \mathrm{~m} \mathrm{~s}^{-1}$. At the position of the $f_{\mathrm{w}}$-event this motion stops completely. On the opposite side, the intergranular plasma is at rest during the $f_{\mathrm{w}}$-event. These properties of the $f_{\mathrm{w}}$-event are in agreement with the predictions of numerical simulations made by Steffen \& Freytag (1991), Steffen et al. (1994), Solanki et al. (1996), Steiner et al. (1998), and by Gadun \& Hanslmeier (2000).

From Figs. 1 and 2 we see that the intergranular lanes located at positions $1^{\prime \prime}$ and $4^{\prime \prime}$ that harbour downward motion in the bottom photospheric layers are not co-spatial with the enhancement of the line width, $f_{\mathrm{w}}$, so that the latter is not an immediate consequence of the intergranular downflow. This confirms the conclusion made by Solanki et al. (1996) that observations on the limb do not show an increase of the line width $f_{\mathrm{w}}$ from the downward motion in the intergranular lane.

Both, the spatial extension and the amplitude of the shock signatures are affected by the finite width of the spectral line formation-region, seeing and instrumental effects, and the orientation of the shock front relative to the spectrograph slit. Hence, the measured spatial extent of the $f_{\mathrm{w}}$-signature appears to be larger than the actual width of the shock front, and the enhancement of the line width in the $f_{\mathrm{w}}$-event and the gradient in Doppler velocity across the $f_{\mathrm{w}}$-event is actually larger than detected. Therefore, the actually observed values of these parameters can be much different from the ones predicted from numerical simulations with grid constants much smaller than $0.1^{\prime \prime}$. In order to estimate these effects we have computed synthetic profiles of Fe II $6456.38 \AA$ from a plane-parallel standard solar atmosphere (Vernazza et al. 1981) in which we assume to exist a horizontal, height-independent, constant velocity field $v_{x}(x)$ that abruptly stops at $x=0$, mimicking a shock front, so that $v_{x}(x)=v_{0}$ for $x<0$ and $v_{x}(x)=0$ for $x \geq 0$. The spectral lines are computed along slanted lines-of-sight with $\cos (\theta)=\mu=0.643\left(\theta=50^{\circ}\right)$ so that the line is blue-shifted for $x<0$. Figure 6 shows the line-core Doppler velocity, $v_{\mathrm{d}}$, and the line width, $f_{\mathrm{w}}$, as a function of space for the full spatial resolution of $10 \mathrm{~km}$ (solid line curve) and for a simulated spatial resolution of $0.25^{\prime \prime}$ achieved by a Gaussian blur over individual ray profiles (dashed curve). The dotted curve shows the corresponding value, when we additionally assume that the shock front is not perpendicular to the slit but inclined to it by $45^{\circ}$, which amounts to an additional box-car averaging of $0.5^{\prime \prime}$ width. The value of $v_{0}$ is chosen to be $3 \mathrm{~km} \mathrm{~s}^{-1}$ in which case the "shock front" would move with a velocity $c_{1}-v_{0}<v_{\mathrm{s}}<c_{2}$ to the left (where $c_{1,2}$ is the speed of sound up- and downstream the shock front) consistent with results of 

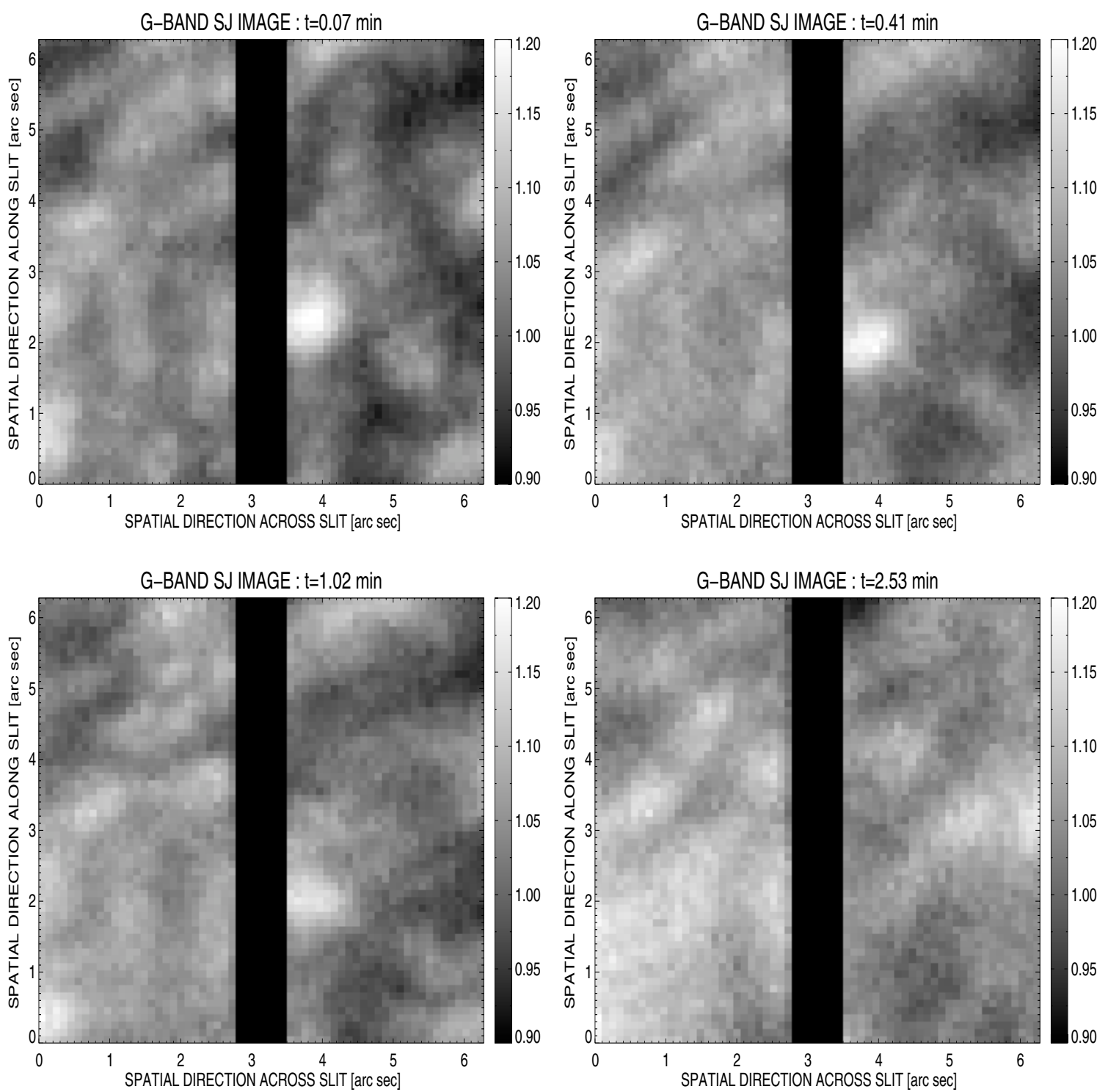

Fig. 4. Evolution of the $G$-band SJ image in the surroundings of the $f_{\mathrm{w}}$-event. The four panels show the temporal changes from the upper left to the bottom right panel with the time information given in their titles. The first three images trace the $G$-band bright point just near the edge of the slit. The last image shows the field after the $f_{\mathrm{w}}$-event has diminished. The position of the slit is marked by the vertical dark rectangle. The $f_{\mathrm{w}}$-event is centred in the middle of the images at $3.3^{\prime \prime}$. The solar limb is directed toward the bottom of the images.

numerical simulations, which show shock front speeds of typically a few $\mathrm{km} \mathrm{s}^{-1}$. First we notice that even in the case of full spatial resolution, the infinitely thin "shock front" results in a finite width of the spectral signature $\left(F W H M\right.$ of $\left.f_{\mathrm{w}}\right)$ of $0.26^{\prime \prime 2}$. Correspondingly, a finite horizontal spatial resolution of only $0.25^{\prime \prime}$ does not dramatically change these values. The maximum value of $f_{\mathrm{w}}$ decreases from 126 to $125 \mathrm{~m} \AA$ and the width of the $f_{\mathrm{w}}$-signal increases from $0.26^{\prime \prime}$ to $0.3^{\prime \prime}$. A substantial decrease of $\max \left(f_{\mathrm{w}}\right)$ to $119 \mathrm{~m} \AA$ and an substantial increase of the

\footnotetext{
${ }^{2}$ This is because of the finite width of the spectral-line formationregion, which translates to a horizontal sampling for an inclined line-of-sight. The depression contribution-functions for the Fe II $6456.38 \AA$ line peak at $\tau_{\mathrm{c}}=0.5$ to $\tau_{\mathrm{c}}=0.05$ when going from line core to the line wing with a spread of more than three decades, so that the full line forms over a region from $\tau_{\mathrm{c}}=2$ to less than $\log \tau_{\mathrm{c}}=-3$.
}

signal width to $0.57^{\prime \prime}$ ensues when inclining the shock front by $45^{\circ}$ with respect to the direction of the slit. Note, that this is an idealized model and that the shape and values of $f_{\mathrm{w}}$ and $v_{\mathrm{d}}$ strongly depend on the actual velocity field and the thermal structure of the atmosphere. However, it gives a good indication for changes due to degradation effects.

Both Fe II lines and also the other medium (Ca I 6455.6 $)$ ), weak (Co I 6455.0 A) and strong (Fe I 4994.11 ^̊) lines display qualitatively very similar temporal and spatial variations of the spectral characteristics. The formation heights of these lines are 110 and $205 \mathrm{~km}$ for the Fe II lines and 170, 40, and $470 \mathrm{~km}$ for Ca I, Co I, and Fe I lines, respectively (see Table 1 for details). This hints at the whole middle and upper photosphere being influenced by the fast motion of the plasma which abruptly stops at the interface from the granulum to the intergranular lane. 


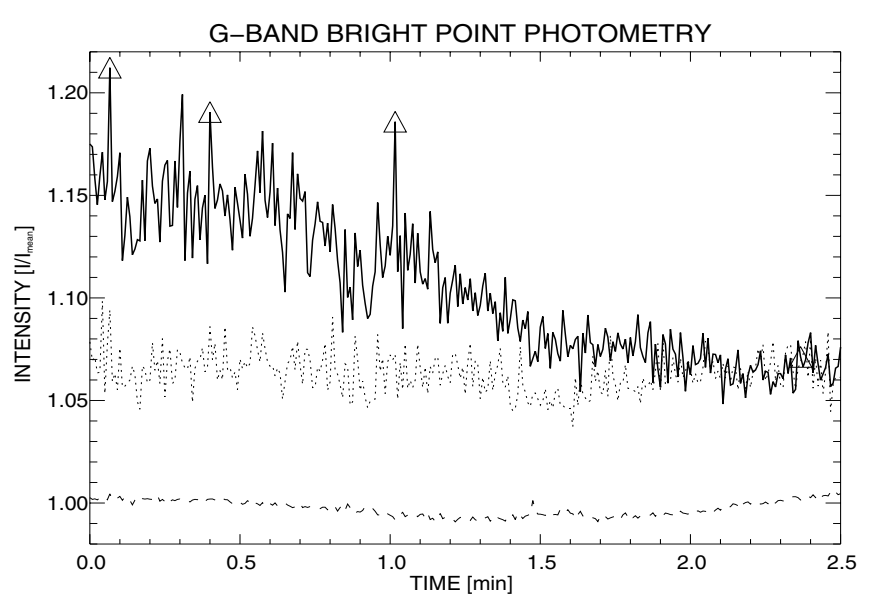

Fig. 5. Temporal evolution of the maximum intensity of the $G$-band bright point. The figure shows the maximum intensity of the bright point (solid line), the maximum intensity of the granular pattern found on the opposite side of the slit (dotted line), and the average intensity of the $G$-band image (dashed line). The triangles mark the first three $G$-band images shown in Fig. 4 .

The $f_{\mathrm{w}}$-events seem to coincide with enhanced non-thermal motion as was put forward by Nesis et al. (1993). These authors found large velocity gradient within a small spatial range just at the borders of granules: not at arbitrary position within the intergranular lane. Different from Nesis et al. (1993), our observations show large velocity gradients in combination with a line width excess near disk limb, which hints at a sharp gradient in the velocity of the (horizontal) flow.

Similar observations at almost the same heliocentric angle were presented by Solanki et al. (1996). Their most significant events show values of the line width excess $(25-35 \%)$, the equivalent width excess $(8 \%)$, and the residual line intesity changes $(20 \%)$ similar to our results. The Doppler velocity differences across the events are smaller (typically up to $600 \mathrm{~m} \mathrm{~s}^{-1}$ ) than in our case. The most extreme profiles of all the lines we have observed show a blue line asymmetry with a bisector shift of up to $-2 \mathrm{~km} \mathrm{~s}^{-1}$. It is in agreement with the asymmetry of the Ni I line found by Solanki et al. (1996). No double profiles were found in the case of our $f_{\mathrm{w}}$-event, unlike to some of the synthesized results presented by Solanki et al. (1996). Nevertheless, the explanation of this phenomenon, originally proposed by Solanki et al. (1996) for a moderate jump in velocity, remains valid for our observations, too: the line absorption is produced by two separated plasma volumes of two different Doppler velocities, for example, by the preshock and postshock medium with one volume of plasma at high (or supersonic) speed with significant blue-shift of a few $\mathrm{km} \mathrm{s}^{-1}$ and the other volume at rest on which this motion is braking.

More than 50 granules of different size and lifetime that were present within the slit length of $123^{\prime \prime}$ (with respect to the real solar surface) were followed for $15 \mathrm{~min}$. For a statistical investigation, normalized line widths, $f_{w, N}$ were calculated dividing every line width, $f_{\mathrm{w}}$, by the mean value of the line width at the corresponding limb distance, which varies between 145 and $150 \mathrm{~m} \AA$. The resulting histogram, shown in Fig. 7, contains all the measurement points of the observed space-time domain

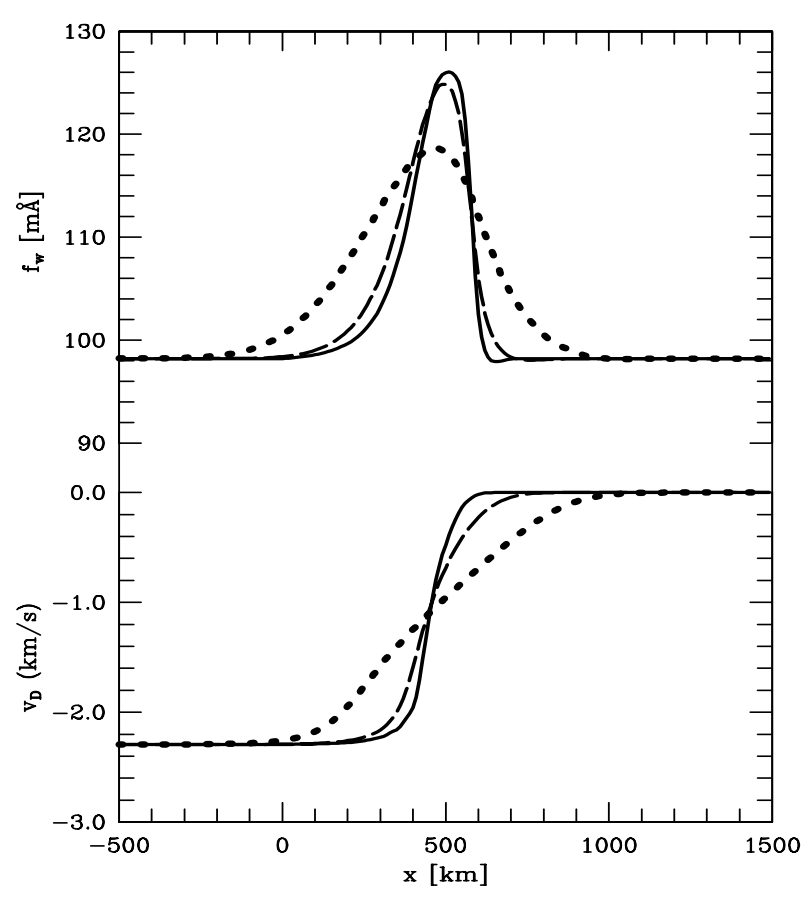

Fig. 6. Spectral signature of a discontinuous flow field with $v_{x}(x)=v_{0}$ for $x<0$ and $v_{x}(x)=0$ for $x \geq 0$ embedded in a plane parallel standard solar atmosphere. The spectral line of Fe II $6456.38 \AA$ is computed along slanted lines-of-sight $(\cos \theta=0.643)$ and corresponding Doppler-shifts (bottom) and FWHM (top) are plotted as a function of the spatial position of the ray entry-point at a height of $800 \mathrm{~km}$ above $\tau_{\mathrm{c}}=1$. The solid curves are obtained with the full spatial resolution of $10 \mathrm{~km}$, the dashed curve when applying a Gaussian blur of $0.25^{\prime \prime}$ width using neighbouring ray profiles, and the dotted curves when applying an additional box-car average of $0.5^{\prime \prime}$ width mimicking a shock front that is inclined by $45^{\circ}$ with respect to the spectrograph slit.

of $123^{\prime \prime} \times 900 \mathrm{~s}$. The distribution of the normalized line widths, $f_{\mathrm{w}, \mathrm{N}}$, consists of two components. The central, main component is nearly Gaussian, positioned at 0.993 and its Gaussian width is 0.122 . The second component, located within a range from $\sim 1.1$ up to 1.3 , is very weak and contains just $0.45 \%$ of all the values that enter the distribution. This component is supposed to be caused by shocks waves because the intergranular lanes do not show any significant line broadening at large viewing angles as was shown by Solanki et al. (1996). The range of values of the second component is in line with the result of the $f_{\mathrm{w}}$ signature derived from the idealized shock model of Fig. 6. Therefore, the fraction of the time-space domain that shows spectral lines affected by a shock wave can be estimated to maximum $0.45 \%$ in the case of our observation. The threshold for the occurence of shocks is found to be $110 \%$ of the mean value at the given $\mu$. The area of the central component surpassing this threshold is $4.24 \%$ of the whole distribution so that the ratio of the second component area to the central one in this range is $\sim 0.11$. In total only 12 events of enhanced $f_{\mathrm{w}}$ above the specified threshold of $110 \%$ were detected. The duration of these events is from 1 to $3 \mathrm{~min}$. Only 2 of these events exceed the threshold of $120 \%$ above which limit the central component does not interfere. Although there is no tool to unambiguously disentangle the events of enhanced 
FWHM f $\mathrm{w}_{\mathrm{w}, \mathrm{N}}$ DISTRIBUTION

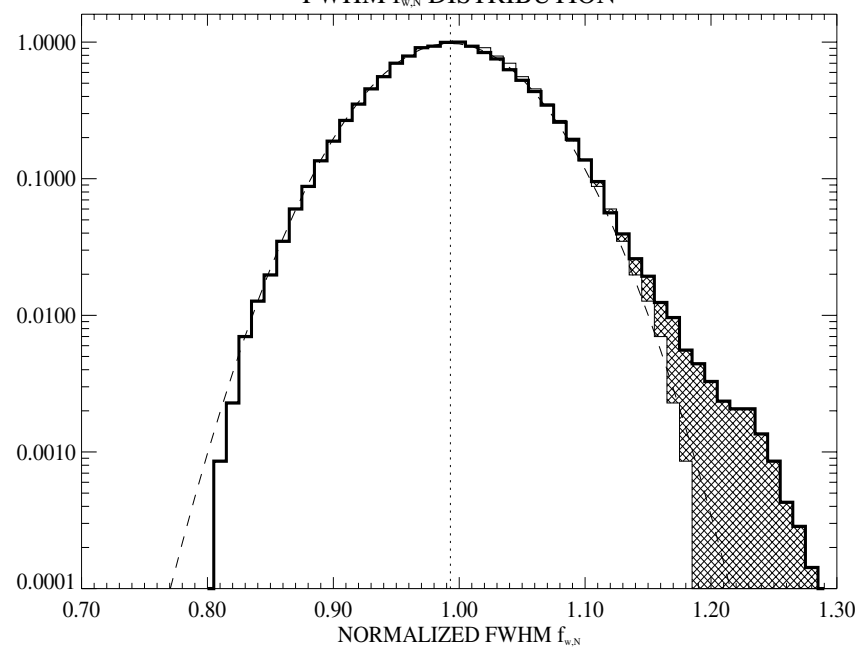

Fig. 7. Histogram of the normalized line widths, $f_{\mathrm{w}, \mathrm{N}}$ (thick solid histogram). The central component of this distribution is fitted by a Gaussian profile (dashed line). The secondary component, representing excess values of $f_{\mathrm{w}, \mathrm{N}}$ (cross-hatched region) was determined as the difference between the positive (right) and the negative (left) part of the distribution. Its fractional area is $0.45 \%$ of the total area of the distribution. The dotted vertical line marks the position of the Gaussian fit for the central component. The histogram includes a total of $18 \times 10^{4}$ values of $f_{\mathrm{w}, \mathrm{N}}$.

$f_{\mathrm{w}}$ within the intermediate range from 110 to $120 \%$, using the statistical approach we can thus estimate an upper limit for the relative occurence of $f_{\mathrm{w}}$-events caused by shocks of $0.45 \%$ of the observed time-space domain.

Supersonic flow (mainly horizontally directed) was predicted to cover an area of $\sim 3-4 \%$ of the solar surface at any one time by numerical hydrodynamic simulations performed by Stein \& Nordlund (1998). This coverage is qualitatively also in agreement with predictions of other numerical simulations (e.g., Cattaneo et al. 1990; Malagoli et al. 1990). However, not all of this area is expected to produce a line broadening but only a fraction pertaining to the shock front, so that the above quoted number can be considered at best an upper limit for the area fraction of $f_{\mathrm{w}}$-events produced by shock waves. In addition, Solanki et al. (1996) show that in order to see a significant shock signature in line profiles the shock front must be viewed close to head-on, which is a rare case in three spatial dimensions. Comparing the occurence of shock signatures $(0.45 \%)$ to the area coverage of supersonic flow predicted by numerical simulations (3-4\%) we can conclude that only at about $10 \%$ of this area shows signs of shock waves.

Several other examples of extremely distorted spectral line profiles have been presented from spatially highly resolved measurements by, e.g., Steffen \& Freytag (1991), Holweger \& Kneer (1989), Wiehr \& Kneer (1988), and Amer \& Kneer (1993). Generally, all these examples represent only single snapshots of spectra and not the temporal evolution of the events.

However we can compare our results with numerical simulations of the temporal evolution of the solar atmosphere presented by Steiner et al. (1998) that explicitly allow for the formation of shock waves. Their video sequences show several shock events from where we can estimate the duration and spatial dimension of the temperature peak in the post-shock medium. In agreement with these predictions our event(s) are of durations of a few minutes, all lasting less than $15 \mathrm{~min}$. The typical horizontal extension of the simulated post-shock temperature enhancements is a few tenth of an arcsecond. This value is in agreement with the spatial extent along the slit of our $f_{\mathrm{w}}$-event of $0.5^{\prime \prime}$ when both, the actual slit width of $0.5^{\prime \prime}$ and the theoretical spatial resolution of the instrument of $0.25^{\prime \prime}$ are taken into account.

Two types of $G$-band bright points have been found in the solar photosphere (Berger \& Title 2001). The first type appears on the edges of bright, rapidly expanding granules and are nonmagnetic in nature. The second one is co-spatial with a magnetic element and is located in intergranular lanes (Muller et al. 2000; Berger \& Title 2001; Langhans et al. 2002). The $G$-band bright point found in our measurements can be interpreted as intergranular as it is not connected to any large, rapidly expanding granule (Fig. 4). Its peak contrast of 1.2 is comparable to the average contrast of $G$-band bright points. The duration of the observed bright point is also in agreement with the average lifetimes of bright points (Berger \& Title 1996).

In an attempt to answer the question on the causal relation between the observed $G$-band bright point found 2.08" away from the $f_{\mathrm{w}}$-event and the $f_{\mathrm{w}}$-event we can summarize the following findings:

- The temporal variation of the line width in the $f_{\mathrm{w}}$-event (Fig. 2, bottom right panel) and the variation of the $G$-band bright point intensity (Fig. 5) are very similar.

- The declining phase of the $G$-band bright point intensity is steeper showing almost no excess relative to the mean granular $G$-band intensity in the surrounding after $1.5 \mathrm{~min}$. It takes another half a minute for the $f_{\mathrm{w}}$-event to diminish.

- The $f_{\mathrm{w}}$-event and the $G$-band bright point are located on opposite sides of a granule.

- No systematic relative motion of these two phenomena was found over the whole duration of the bright point. The standard deviation from their average distance is relatively large $\left(0.25^{\prime \prime}\right)$, where deviations are caused by randomly distributed, seeing motion.

These findings favour a causal relation between the magnetic field concentration, displayed by the $G$-band bright point, and the selected $f_{\mathrm{w}}$-event. However, the simultaneous existence of the magnetic field concentration and the line-width event being just a coincidence cannot be definitely ruled out. Therefore, the present $f_{\mathrm{w}}$-event could be caused either by the deceleration of the fast granular flow on the edge of the granule, as predicted by Cattaneo et al. (1989), or by the swaying motion or a rapid footpoint motion of the flux concentration, that can lead to a bow shock as seen in the simulations by Steiner et al. (1998), categorized as type II shocks.

Using the 12 identified events of enhanced line width the average lifetime of the events was found to be $2 \mathrm{~min}$. We can estimate that one event can be expected in a time-space domain of roughly $150 \operatorname{arcsec} \times \min$ when the total duration of the observation, the slit length with respect to the real surface, and the 
number of identified events are taken into account. Additional events may have escaped detection because they were below the threshold of $110 \%$ of the mean value of the line width at the given $\mu$. Moreover, this estimation of the spatiotemporal frequency of enhanced $f_{\mathrm{w}}$ phenomena cannot be translated directly into a probability for detecting a shock signature as some of these phenomena may have a physical origin, different from the only event discussed here.

It should be emphasized that performing this particular type of studies is only possible with an extremely high spatial resolution. On-line corrections of image distortions (Schmidt \& Kentischer 1995; von der Lühe et al. 2003) are necessary to perform successful measurements of the temporal evolution of shock signatures in the solar photosphere. In particular, large image field corrections are needed considering the sparse occurence of significant shock signatures. In our case the observed event was quite far from the subfield corrected by the AO setup, which caused some periods of reduced image quality (Fig. 1). Dual-conjugated AO (Berkefeld et al. 2003) may help to overcome these problems in future.

\section{Conclusion}

We have observed variations of spectral line characteristics and changes of the line profiles that are, when compared with numerical simulations of convection in the solar granulation, further indications for the existence of hydrodynamic shocks in the solar photosphere. Agreement was also found with respect to the amplitudes of the spectral characteristics during the event when seeing and instrumental effects are taken into account. These results are in line with the findings of Solanki et al. (1996).

Both, the stable and the declining phases of the shock event were followed for the first time. A possible relation of the shock event with a nearby $G$-band bright point was investigated, indicating a causal relationship. A final conclusion whether the fast flow and associated discontinuity (shock) are caused by either the swaying motion of the flux concentration or by the granular flow not related to the magnetic field cannot definitely be made. The temporal and spatial coincidence of both features suggests that the flow and shock is a consequence of the swaying or random motion of the magnetic flux concentration, while the spatial coincidence of the line-broadening event with the edge of a granule favours interpretation in terms of a shock that was solely generated by the granular flow.

Acknowledgements. The VTT is operated by the Kiepenheuer-Institut für Sonnenphysik, Freiburg, at the Observatorio del Teide of the Instituto de Astrofísica de Canarias. We would like to thank M. Sigwarth and T. Berkefeld for the operation of the AO during our campaign and K. Langhans for his help with the SJ DALSA camera. We thank the referee whose comments greatly helped us to improve the paper. This work was supported by the Slovak grant agency VEGA (2/3015/23) and by the Deutsche Forschungsgemeinschaft grant (DFG 436 SLK 113/7). This research is part of the European Solar Magnetism Network (EC/RTN contract HPRN-CT-2002-00313). A.H., A.K. and J.R. thank the Austrian and Slovak Academies of Sciences for financing the exchange of scientists. A.H. acknowledges the support of the Austrian Fonds zur
Förderung der Wissenschaftlichen Forschung. Finally we would like to acknowledge our late colleague A.S. Gadun who originally initiated this project attracting our interest to this topic.

\section{References}

Allende Prieto, C., \& Garciá López, R. J. 1998, A\&A, 131, 431

Amer, M. A., \& Kneer, F. 1993, A\&A, 273, 304

Berkefeld, T., Soltau, D., \& Lühe, O. von der 2002, in Adaptive Optics Systems Technologies, ed. P.L. Wizinowich, \& D. Bonaccini, Proc. SPIE, 4839, 544

Berger, T. E., \& Title, A. M. 1996, ApJ, 463, 365

Berger, T. E., \& Title, A. M. 2001, ApJ, 553, 449

Cattaneo, F., Hulburt, N. E., \& Toomre, J. 1989, in Stellar and Solar Granulation, ed. R. J. Rutten, \& G. Severino (Dordrecht: Kluwer), 415

Cattaneo, F., Hulburt, N. E., \& Toomre, J. 1990, ApJ, 349, L63

Cattaneo, F. 1999, ApJ, 515, L39

Deinzer, W., Hensler, G., Schüssler, M., \& Weisshaar, E. 1984a, A\&A, 139,426

Deinzer, W., Hensler, G., Schüssler, M., \& Weisshaar, E. 1984b, A\&A, 139,435

Emonet, T., \& Cattaneo, F. 2001, ApJ, 560, L197

Fox, P. A., Theobald, M. L., \& Sofia, S. 1999, ApJ, 383, 860

Gadun, A. S. 2000, private communication

Gadun, A. S., \& Hanslmeier, A. 2000, Kinematika i Fizika Nebesnykh Tel, 16, 130

Gadun, A. S., Solanki, S. K., \& Johannesson, A. 1999, A\&A, 350, 1018

Gray, D. F. 1976, The Observation and Analysis of Stellar Atmosheres (New York: Wiley \& Sons)

Grossman-Doerth, U., Schüssler, M., \& Solanki, S. K. 1988, A\&A, 206, L37

Grossman-Doerth, U., Knölker, M., Schüssler, M., \& Solanki, S. K. 1994, A\&A, 285, 648

Holweger, H., \& Kneer, F. 1989, in Stellar and Solar Granulation, ed. R. J. Rutten, \& G. Severino (Dordrecht: Kluwer), 173

Hurlburt, N. E., \& Toomre, J. 1988, ApJ, 327, 920

Kentischer, T. J. 1995, A\&AS, 109, 553

Knölker, M., Schüssler, M., \& Weisshaar, E. 1988, A\&A, 194, 257

Kučera, A., Rybák, J., Hanslmeier, A., \& Wöhl, H. 2003, Hvar Observatory Bulletin, 27, 25

Kurucz, R. L. 1990, Trans. IAU, XXB, 168 (CD-ROM 23)

Langhans, K. 2000, private communication

Langhans, K., Schmidt, W., \& Tritschler, A. 2002, A\&A, 394, 1069

Lühe, O., von der, Soltau, D., Berkefeld, T., \& Schelenz, T. 2003, in Innovative Telescopes and Instrumentation for Solar Astrophysics, ed. S. L. Keil, \& S. V. Avakyan, Proc. SPIE, 4853, 187

Malagoli, A., Cattaneo, F., \& Brummell, N.H. 1990, ApJ, 361, L33

Maltby, P., Avrett, E. H., Carlsson, M., et al. 1986, ApJ, 306,284

Moore, C. E. 1972, A Multiplet Table of Astrophysical Interest, NSRDS-NBS 40 (Washington: NBS)

Moore, C. E., Minnaert, M. G. J., \& Houtgast, J. 1966, The Solar Spectrum $2935 \AA$ to $8770 \AA$ A, NBS Monograph, 61 (Washington: NBS)

Muller, R., Dollfus, A., Montagne, M., Moity, J., \& Vigneau, J. 2000, A\&A, 359, 373

Neckel, H. 1999, Sol. Phys., 184, 421

Nesis, A., Bogdan, T. J., Cattaneo, F., et al. 1992, ApJ, 399, L99

Nesis, A., Hanslmeier, A., Hammer, R., et al. 1993, A\&A, 279, 599

Nordlund, A. 1984, in Small-Scale Dynamical Processes in Quiet Stellar Atmospheres, ed. S. L. Keil (Sunspot, NM, USA), 181 
Nordlund, Å. 1986, in Small Scale Magntic Flux Concentrations in the Solar Photosphere, ed. W. Deinzer, M. Knölker, \& H. Voigt (Göttingen: Vandenhoeck \& Ruprecht), 83

Ploner, S. R. O., Solanki, S. K., \& Gadun, A. S. 1999, A\&A, 352, 679

Rimmele, T., Dunn, R., Richards, K., Radick, R. 1999, in High Resolution Solar Physics: Theory, Observations, and Techniques, ed. T. R. Rimmele, K. S. Balasubramaniam, \& R. R. Radick, ASP Conf. Ser., 183, 222

Schmidt, W., \& Kentischer, T. 1995, A\&AS, 113, 363

Schröter, E. H., Soltau, D., \& Wiehr, E. 1885, Vist. Astron., 28, 519

Solanki, S. K., \& Stenflo, J. O. 1985, A\&A, 148, 123

Solanki, S. K., Rüedi, I., Bianda, M., \& Steffen, M. 1996, A\&A, 308, 623

Steffen, M., Ludwig, H.-G., \& Krüss, A. 1989, A\&A, 213, 371

Steffen, M., \& Freytag, B. 1991, Rev. Mod. Astron., 4, 43

Steffen, M., Freytag, B., \& Holweger, H. 1994, in Solar Magnetic Fields, ed. M. Schüssler, \& W. Schmidt (Cambridge: Cambridge University Press), 298
Stein, R. F., \& Nordlund, Å. 1989, ApJ, 342, L95

Stein, R. F., \& Nordlund, Å. 1998, ApJ, 499, 914

Stein, R. F., \& Nordlund, A. 2002, in Magnetic Coupling of the Solar Atmosphere Euroconference and IAU Colloquium 188, ed. H. Sawaya-Lacoste, ESA SP-505 (Noordwijk, Netherlands), 83

Steiner, O., Grossman-Doerth, U., Knölker, M., \& Schüssler, M. 1994, in Solar Magnetic Fields, ed. M. Schüssler, \& W. Schmidt (Cambridge: Cambridge University Press), 282

Steiner, O., Grossman-Doerth, U., Knölker, M., \& Schüssler, M. 1998, ApJ, 495, 468

Vernazza, J. E., Avrett, E. H., \& Loeser, R. 1981, ApJS, 45, 635

Vögler, A., \& Schüssler, M. 2003, Astron. Nachr. 324, 399

Weiss, N. O., Brownjohn, D. P., Matthews, P. C., \& Proctor, M. R. E. 1996, MNRAS, 283, 1153

Wiehr, E., \& Kneer, F. 1988, A\&A, 195, 310

Wöhl, H., Kučera, A., Rybák, J., \& Hanslmeier, A. 2002, A\&A, 394, 1077 